\title{
El aprendizaje de los conceptos genéricos entre niños quechuahablantes monolingües
}

L'apprentissage des concepts génériques parmi les enfants quechuaphones monolingues

Learning generic concepts among monolingual Quechuaphone children

Bruce Mannheim y Susan A. Gelman

\section{(2) OpenEdition}

Journals

Edición electrónica

URL: http://journals.openedition.org/bifea/4132

DOI: $10.4000 /$ bifea.4132

ISSN: 2076-5827

Editor

Institut Français d'Études Andines

Edición impresa

Fecha de publicación: 1 diciembre 2013

Paginación: 353-368

ISSN: 0303-7495

Referencia electrónica

Bruce Mannheim y Susan A. Gelman, «El aprendizaje de los conceptos genéricos entre niños quechuahablantes monolingües », Bulletin de l'Institut français d'études andines [En línea], 42 (3) | 2013, Publicado el 08 diciembre 2013, consultado el 05 noviembre 2020. URL : http:// journals.openedition.org/bifea/4132; DOI : https://doi.org/10.4000/bifea.4132

\section{(c) (i) $\odot$}

Les contenus du Bulletin de l'Institut français d'études andines sont mis à disposition selon les termes de la licence Creative Commons Attribution - Pas d'Utilisation Commerciale - Pas de Modification 4.0 International. 


\title{
El aprendizaje de los conceptos genéricos entre niños quechuahablantes monolingües*
}

\author{
Bruce Mannheim \\ Susan A. Gelman**
}

\section{Resumen}

Las expresions genéricas desempeñan un papel crítico en el desarrollo conceptual de los niños quechuahablantes monolingües de la parte sur del Perú. Este artículo aborda diferentes teorías sobre la adquisición y el desarrollo conceptual de los niños de temprana edad, elaborando una caracterización de conceptos genéricos que se refieren a categorías abstractas. Profundiza la manera cómo estos se expresan en quechua, exponiendo los datos de una serie de investigaciones sobre la adquisición de conceptos genéricos entre niños quechuahablantes. Los resultados confirman que estos diferencian los enunciados genéricos de los específicos así como de los cuantificadores. Coinciden con los resultados obtenidos con hablantes de inglés y de mandarín. Se observan notables correlaciones en la interpretación de los conceptos genéricos por parte de los hablantes de distintas lenguas a través del mundo. Otro resultado sobresaliente es el hecho de que los niños quechuahablantes atribuyen un valor genérico a los enunciados que lingüísticamente están no-marcados. La capacidad universal de conceptualizar está relacionada con contextos culturales y lingüísticos particulares en la adquisición y el desarrollo de los conceptos. Las construcciones genéricas, aunque no tengan marcas morfológicas, constituyen un elemento esencial de articulación entre los procesos cognitivos universales y las particularidades de las lenguas y culturas, la matriz y la ontología específica del quechua.

Palabras clave: niños quechuahablantes, conceptos, expresiones genéricas, desarrollo cognitivo, Perú

* La investigación fue conducida con el apoyo de la beca NICHD HD36043. Agradecemos nuestros colaboradores en la investigación mencionados en este artículo: Carmen Escalante, Margarita Huayhua y Rosalía Puma. Asimismo agradecemos a los papás y niños que participaron en la investigación, al Centro Bartolomé de las Casas en Cuzco por su apoyo administrativo, a Guillermo Salas Carreño por su ayuda con las traducciones de los stumuli quechua así como a Felicia Kleinberg, Erin Boyle y Allison Wachter por su ayuda en entrar los datos. También agradecemos a Palmira La Riva González por su gentileza en la traducción del texto al castellano. La traducción final fue revisada por los autores. En algunas instancias hay términos técnicos que no tienen una traducción generalmente aceptada en castellano. En estos casos se ha mantenido el término original después, entre paréntesis.

**University of Michigan. E-mails: mannheim@umich.edu; gelman@umich.edu 


\title{
L'apprentissage des concepts génériques parmi les enfants quechuaphones monolingues
}

\section{Résumé}

Les expressions génériques jouent un rôle critique dans le développement conceptuel des enfants quechuaphones monolingues du sud du Pérou. Cet article aborde différentes théories sur l'aquisition et le développement conceptuel des enfants en bas âge et élabore une caractérisation des concepts génériques qui font référence à des catégories abstraites. II approfondit la manière dont ceux-ci sont exprimés en quechua par l'exposé de données d'une série d'enquêtes sur l'acquisition des concepts génériques parmi des enfants quechuaphones. Les résultats confirment que ces derniers différencient les énoncés génériques des énoncés spécifiques ainsi que des énoncés quantificateurs. Ils coincident avec les résultats obtenus auprès d'anglophones et sinophones. Des correlations notables s'observent dans l'interprétation des concepts génériques de la part des locuteurs de différentes langues à travers le monde. Un autre résultat important est le fait que les enfants quechuaphones attribuent une valeur générique aux énoncés qui sont linguistiquement non marqués. La capacité universelle de conceptualiser est liée à des contextes culturels et linguistiques particuliers dans l'acquisition et le développement des concepts. Les constructions génériques, quoique ne possédant pas de marques morphologiques, constituent un élément essentiel d'articulation entre les processus cognitifs universels et les particularités des langues et cultures, la matrice et l'ontologie spécifique du quechua.

Mots-clés : enfants quechuaphones, concepts, expressions génériques, développement congnitif, Pérou

\section{Learning generic concepts among monolingual Quechuaphone children}

\begin{abstract}
Generic expressions play a critical role in the conceptual development of monolingual Southern Peru Quechua children. This article discusses different theories about aquisition and conceptual development of young children and makes a characterization of generic concepts referring to abstract categories. It deepens how these are expressed in Quechua by the presentation of data from a series of surveys on the acquisition of generic concepts among Quechuaphone children. Results confirm that they differentiate generics from specific utterances as well as from quantifiers. They coincide with the results obtained with English and Mandarin speakers. Significant correlations are observed in the interpretation of generic concepts by speakers of worldwide different languages. Another important result is that Quechuaphone children assign generic interpretations to linguistically unmarked utterances. Universal conceptual capacities interact with particular cultural and linguistic contexts to guide conceptual development. Generic expressions — though unmarked morphologically — are one important point of contact between general cognitive processes and the particularities of languages and cultures, the matrix of a specifically Quechua conceptual framework and ontology.
\end{abstract}

Keywords: quechuaphone children, concepts, generic expressions, cognitive developement, Peru

\section{INTRODUCCIÓN}

Los seres humanos se enfrentan con el problema universal de representar las experiencias de la vida cotidiana de manera eficiente, de su generalización a nuevos contextos y la forma de promover la comunicación con los otros. En otros 
términos, se enfrentan con el problema de la formación y del desarrollo de los conceptos. Tomando en cuenta que estos constituyen la base para el pensamiento humano, es necesario examinar desde el punto de vista del desarrollo cognitivo la manera como estos se elaboran y se modifican. En este artículo abordamos diferentes teorías sobre la adquisición y el desarrollo conceptual temprano, elaborando una caracterización de conceptos genéricos (los conceptos que hacen referencia a categorías abstractas). Se profundiza la manera como estos se expresan en quechua exponiendo los resultados de una serie de investigaciones empíricas sobre la adquisición de conceptos genéricos en niños quechuahablantes. Sugerimos que la capacidad universal de conceptualizar está en correlación con contextos culturales y lingüísticos particulares en la adquisición y el desarrollo de conceptos. Sobre la base de las evidencias empíricas del desarrollo cognitivo de los niños monolingües quechuahablantes preescolares, es indiscutible el hecho que ellos mismos usan conceptos generales, tal como los niños de cualquier otra cultura.

\section{CONCEPTOS COMO PRIMITIVOS VERSUS CONCEPTOS EN TEORÍAS}

Los orígenes de la formación de los conceptos han sido debatidos por diversos autores durante milenios (cf. la discusión de Sócrates en el Menón). Entre las diferentes perspectivas teóricas podemos distinguir dos posiciones principales que denominaremos el enfoque de los «conceptos como primitivos» y el de los «conceptos en teorías». El primero supone que la elaboración de conceptos está ligada directamente a los fenómenos de la experiencia. Desde este punto de vista, los niños adquirirían el concepto de «perro» a partir de la experiencia perceptiva de un perro en particular, del registro de dicha experiencia y por último, de su generalización a otros contextos. Esta teoría postula que la formación de los conceptos tiene su origen en la percepción y está vinculada a eventos particulares. Cada concepto es una unidad «atómica» -indivisible y autónoma-. Keil et al. (1998: 104) consideran que esta perspectiva supone intrínsecamente dos presupuestos o «dogmas»:

1) «Toda categoría nueva es inicialmente comprendida por medio de los mecanismos de similitud y asociación (procesamiento de la similitud) y solo posteriormente por medio de la consideración de los principios causales y explicativos». Estos últimos solo se llevarían a cabo cuando se cuenta con un mayor grado de desarrollo cognitivo.

2) «En una primera instancia, los infantes y niños utilizarían la dimensión asociativa de los conceptos para representar las categorías. Solo con el desarrollo cognitivo emerge una explicación más abstracta de los conceptos».

En contraste con esta teoría, la perspectiva de los «conceptos en teorías», postula que estos emergen a partir de conocimientos más generales y amplios que tienen los niños sobre el mundo. Estas teorías implícitas o de «sentido común» comparten con las teorías científicas el hecho de establecer ontologías de dominio específico, 
leyes causales de dominios específicos y entidades inobservables de dominios específicos (Carey, 1985). Sin embargo, estas teorías difieren de las teorías científicas por ser menos sistemáticas, menos precisas y al no estar sujetas a un control científico riguroso. Según Rochel Gelman (Gelman \& Williams, 1998), Simons \& Keil (1995), Wellman \& Gelman (1998), y otros autores, infantes y niños pequeños poseen una estructura cognitiva de base que les permite una clasificación rápida y el aprendizaje de nueva información. Desde este punto de vista, la adquisición temprana de conceptos no es estrictamente de origen perceptivo, sino que se encuentra relacionada con configuraciones ontológicas más amplias (ej.: animado/inanimado) y con expectativas respecto a las leyes causales de las que los conceptos forman parte (ej.: un perro es clasificado inicialmente como un ser animado, un agente capaz de realizar un movimiento de manera autónoma). De manera relevante, las teorías «ingenuas» (tacitas, naives) o de «sentido común» tienen la particularidad de ser teorías de dominio específico (ej. teoría de la mente (theory of mind), teoría de la biología, teoría del mundo físico). Dichas teorías se sirven de las regularidades estadísticas del input para la adquisición y el procesamiento de nuevas estructuras conceptuales (Xu \& Kushnir, 2013).

Si bien es indiscutible que los niños elaboran los conceptos tanto a partir de la inducción (de lo particular a lo general) como de la deducción (de lo general a lo particular) (Waxman \& Gelman, 2010), estamos convencidos del rol esencial que juegan los complejos teóricos en la adquisición y el desarrollo temprano de conceptos en los niños. Una confirmación de la validez del punto de vista de «conceptos en teorías» es el hecho de que la formación y el desarrollo de conceptos en los niños pequeños van mucho más allá de las evidencias perceptibles (Gelman \& Markman, 1986; Gelman \& Wellman, 1991; Gelman, 2003; Massey \& Gelman, 1988; Hauf et al., 2012). Consideramos así que los niños poseen primero expectativas más amplias respecto a los conceptos que expectativas específicas (las expectativas más generales preceden a las expectativas más específicas) (Simons \& Keil, 1995), que sus creencias están interrelacionadas (Slaughter \& Gopnik, 1996) y que las distinciones ontológicas influyen de manera decisiva en sus inferencias (Opfer \& Gelman, 2010). Mientras que teorías previas sobre la formación y el desarrollo temprano de conceptos asumían la posición que considera los «conceptos como primitivos» de manera axiomática. A partir de los años 1980, diversos investigadores han acumulado evidencias experimentales que atestiguan que los conceptos se forman y desarrollan al interior de teorías de dominio específico. No obstante, el debate en torno a esta problemática continúa de manera intensa.

\section{CONCEPTOS GENÉRICOS}

Los conceptos se pueden clasificar en específicos (mamá, esta cucharilla, mi llama) y genéricos (mujeres, cucharillas, llamas). En este artículo, nos interesamos específicamente en los conceptos generales expresados lingüísticamente con frases nominales genéricas (ej. el pájaro vuela). Las frases nominales genéricas se 
distinguen porque hacen referencia a categorías abstractas, distinguiéndose así de los enunciados específicos (ej.: estos pájaros están volando). El aprendizaje de las frases nominales genéricas plantea varios retos al aprendiz:

- las categorías a las que hacen referencia son abstractas (ej.: la categoría de pájaro: pasado, presente, futuro e hipótesis) y por lo tanto no pueden ser percibidas o evidenciadas;

- el significado de las frases genéricas no puede reducirse a una regla simple (ej.: algunas proposiciones inusuales pueden expresarse por medio de frases genéricas [los mosquitos vehiculan el virus del Nilo Occidental], sin embargo generalmente no sucede así [la gente es diestra] [Leslie, 2008]);

- hasta ahora no se ha encontrado ninguna lengua que tenga marcadores específicos para las proposiciones genéricas, y contrariamente a los cuantificadores (todos, algunos, la mayoría), los genéricos solo se pueden inferir a partir del contexto (este aspecto será desarrollado en la siguiente sección).

Por todas estas razones las frases genéricas plantean un desafío a los aprendices. En efecto, no hay un referente concreto al que referirse, ni una regla unívoca en la que uno se pueda guiar y para concluir, ningún marcador lingüístico específico que las identifique. Sin embargo, estudios previos han demostrado que niños de lengua inglesa de 2 a 3 años de edad, están en la capacidad de generar y comprender el lenguaje genérico (Gelman, 2003).

\section{LOS ENUNCIADOS GENÉRICOS EN QUECHUA}

Todas las lenguas parecieran disponer de recursos lingüísticos para formar oraciones genéricas. Sin embargo, estos recursos varían según los idiomas. En inglés, por ejemplo, las construcciones genéricas se expresan por medio de los plurales simples (sin determinante o cuantificador antepuesto al sustantivo), por ejemplo «Birds fly» (pájaros vuelan), mientras que en español el determinante antepuesto al nombre es necesario para formar las construcciones genéricas (ej.: «El pájaro vuela»), en tanto que la pluralización del sustantivo le da un carácter específico y no genérico (ej.: «los pájaros vuelan» hace referencia a pájaros específicos).

La mayoría de las investigaciones previas sobre la adquisición y el desarrollo de los conceptos genéricos se han concentrado en los niños preescolares de habla inglesa (Cimpian \& Markman, 2008; 2009; Gelman, 2004) y algunas investigaciones han examinado la adquisición y el desarrollo temprano de conceptos genéricos en niños que aprenden el mandarín (Tardif et al., 2012). Tanto la lengua inglesa como el mandarín tienen una morfología hasta cierto punto limitada. Mientras que el quechua (tanto la variante sur peruana como las otras variantes dialectales) es altamente flexivo, los genéricos (conceptos, oraciones, formas) se caracterizan por la ausencia de marcadores lingüísticos. Es así que esta lengua difiere estructuralmente de aquellas en las que el uso de lenguaje genérico ha sido estudiado hasta ahora (el inglés y el mandarín). El quechua es una lengua aglutinante de tipo SOV (sujeto-objeto-verbo) o de tipo III según la tipología lingüística establecida por 
Greenberg (1963). Es una lengua altamente sintética de tipo OV con hasta 3 o 4 sufijos nominales y de 5 a 10 sufijos verbales. En este contexto de complejidad gramatical, los genéricos no están marcados morfológicamente, caracterizándose precisamente por la falta de marcadores específicos (sobre todo de tiempos y aspectos verbales). Las oraciones siguientes ilustran el contraste entre oraciones genéricas y no genéricas en quechua:

(i)

Waka q'achuta mihun.

Waka q'achu-ta mihu-n.

El ganado vacuno pasto -acc. come-3

El ganado vacuno come pasto

(ii)

Wakaqa q'achutan mihushan watananpi.

Waka-qa q'achu-ta-n mihu-sha-n wata-na-n-pi.

El vacuno-topicalizador pasto-acc-evidencial come-progresivo -3

atar-n ominalizador-3 poss-loc.

La vaca está comiendo pasto en su corral (amarradero, estaca)

La oración (i) es genérica ya que hace referencia al vacuno en general y no a una vaca o toro en particular. Notamos que en estas oraciones la presencia de marcadores morfológicos es reducida —el término waka 'ganado vacuno' está en su forma simple, no lleva ninguna marca que indique su número-; q'achu 'pasto' tiene solo la marca de ser el objeto (por el acusativo, -ta); y mihu- 'comer' está conjugado en la tercera persona (singular) sin marcadores de tiempo, aspecto o número. En cuanto a la oración (ii) (de Cusihuamán Gutiérrez, 1976: 238) esta no es genérica ya que se refiere a una vaca específica atada a una estaca (en un corral). Esta oración lleva la marca del sufijo -mi (en este caso el alomorfo después de una vocal, $-n)$, haciendo q'achu-ta 'pasto' el foco pragmático de la oración. La frontera izquierda del ámbito del foco pragmático está marcado por -qa en el sujeto gramatical waka. El verbo mihu- 'comer' lleva la marca del durativo, indicando que la acción está en curso de ser realizada (marcando por lo tanto el aspecto, aunque no así el tiempo verbal). El carácter particular del ganado vacuno se indica no solo al señalar que está atado a un amarradero, sino también con el uso de los morfemas gramaticales (en especifico -sha, -mi, and -qa), incluso si waka no constituye el foco de la oración.

La formulación de expresiones genéricas en quechua, ilustradas con los ejemplos precedentes, contrasta con la expresión de oraciones genéricas en inglés o en español. En inglés, existen dos formas gramaticales pertinentes para distinguir si una oración es genérica o no: el definido versus el indefinido - expresado por el uso de artículos- y plural versus singular (número). Por ejemplo, la oración Barns are red es genérica, y se refiere a barns (graneros) como categoría, mientras que las frases The barns are red o The barn is red no son genéricas ya que hacen referencia a uno o más graneros en particular (Véase Carlson \& Pelletier, 1995; Gelman, 2004, para un análisis detallado sobre las proposiciones genéricas en inglés, incluyendo la función del verbo y el contexto pragmático). Tomando en cuenta que la lengua española exige la concordancia de género en la frase 
nominal, no se puede usar el plural sin un artículo precedente (el llamado «bare plural») para expresar lo genérico. Frente a la ausencia de un marcador en la oración, las frases nominales — singular como plural— expresan una ambigüedad respecto a su carácter genérico o específico, como en los ejemplos (iii)-(iv). En español, tanto el contexto sintáctico como el discursivo, incluyendo el foco y el orden de las palabras, juegan un rol fundamental en la interpretación de las frases nominales como genéricas o específicas, muy parecido al inglés o mandarín, pero sin la posibilidad de usar radicales simples.

(iii) el perro ladra (los perros ladran en general o un perro específico se caracteriza por ladrar),

(iv) los perros ladran (los perros ladran en general o unos perros específicos ladran),

Si el sujeto de la frase nominal se desplaza del foco se favorece la interpretación genérica:

(iiia) ladra el perro,

(iva) ladran los perros.

En cuanto a la gramática quechua, no se marca la distinción entre el definido y el indefinido, y la marca del número no es obligatoria. Eso quiere decir que se puede expresar la pluralidad, pero la ausencia del pluralizador (-kuna) no implica que el sujeto sea singular obligatoriamente; tampoco se requiere ni la concordancia verbal ni la nominal. Desde el punto de vista de la sintaxis, no existe una sola construcción genérica; en quechua esta se caracteriza por la ausencia de especificidad gramatical. La interpretación genérica es la condición base (default) en las frases nominales quechuas. A título de comparación, vale señalar que en quechua como en inglés y en mandarín, no hay marca morfológica de género en las frases nominales. En cuanto que en español y en francés, el género está implícito en el artículo (el determinador), así el equivalente en estas lenguas al radical simple en inglés y mandarín para marcar el género, es un sustantivo precedido del artículo.

Tomando en cuenta que la lengua quechua es de tipo aglutinante y altamente inflexionada, esta constituye un caso ideal para examinar la proposición que sostiene que las expresiones genéricas resultan de las interpretaciones de base en los niños en proceso de adquisición del lenguaje (véase Chierchia, 1998, para una proposición similar). Si los niños aprenden el uso de las expresiones genéricas mediante la presencia de los pies lingüísticos, entonces el quechua debería resultar una lengua extremamente difícil para los niños en cuestión de aprender las formas genéricas, ya que estas no tienen marcas morfológicas específicas que las identifique. En efecto, el quechua no incluye en la frase nominal un marcador obligatorio para expresar su dimensión genérica (ej.: plurales o determinantes). Por otra parte, si los niños distinguen las proposiciones genéricas por medio de la ausencia de marcadores de la especificidad, entonces el quechua debería resultar particularmente fácil, ya que esta lengua se caracteriza por ser altamente inflexionada y marca de diversas maneras la especificidad, incluyendo algunas 
maneras que no existen en el español, lo que se puede apreciar en los ejemplos expuestos más abajo. Las partes relevantes en quechua están en cursivas y las traducciones al castellano en paréntesis. Por ejemplo, en castellano el tiempo verbal indica especificidad ya que sitúa un evento en un momento temporal específico (i.e.: pasado); el aspecto indica especificidad ya que de igual manera sitúa un fenómeno en un periodo de tiempo particular (ej. durativo). El uso de evidenciales en quechua indica si la relación entre el hablante y una acción determinada y por lo tanto no puede tratarse de una proposición genérica1. En cambio, la ausencia de estos marcadores indica - a los adultos - su carácter genérico:

(v)

Wakaqa q'achutas mihuran.

Dice que (evidencial) la vaca se comió el pasto. (evidencial + tiempo verbal)

(vi) Wakaqa q'achutan mihuran.

Fío que (evidencial) la vaca se comió el pasto. (evidencial + tiempo verbal)

(vii) Wakaqa q'achutas mihushan.

Dice que (evidencial) la vaca está comiendo el pasto. (evidencial + durativo)

(viii) Wakaqa q'achutans mihushan.

Fío que (evidencial) la vaca está comiéndose el pasto. (evidencial + durativo)

(ix) Waka q'achuta mihunman.

La vaca podría comerse el pasto (condicional)

Una motivación adicional para el estudio de los genéricos en quechua se debe al hecho que el contexto cultural inmediato de los pobladores quechuahablantes difiere de forma contrastante del ámbito sociocultural en el que se han desarrollado hasta ahora los estudios sobre las proposiciones genéricas, caracterizado por ser un contexto de clase media altamente instruida. El uso de los enunciados genéricos ha estado asociado a ámbitos pedagógicos, incluyendo contextos de lectura de libros (Gelman \& Tardif, 1998) y de secuencias de enseñanza implícita (Csibra \& Gergely, 2009; Gelman et al., 2013). De este modo, en los Estados Unidos, el uso de las formas genéricas se ve privilegiado en los videos informativos, en los programas televisivos, en los libros de ensayo y en el contexto académico. Poseemos muy poca información sobre la manera cómo los niños adquieren e utilizan las frases genéricas en contextos culturales donde predomina la educación informal, con menos presencia de libros.

1 Los sufijos evidenciales pueden también marcar una afirmación personal a la autoridad por parte del hablante (Faller, 2002), siempre cuando su oración depende del conocimiento enciclopédico en vez del conocimiento personal. 
Podemos empezar a explorar las variaciones culturales del uso de las oraciones genéricas examinando el lenguaje genérico de los niños quechuahablantes preescolares monolingües en el contexto rural con una economía agropastoral en que se llevó a cabo esta investigación. La comunidad que participó en esta investigación se encuentra en la provincia de Calca, departamento del Cusco. Esta comunidad tiene acceso tanto a las tierras bajas donde siembran maíz y granos como a una zona de puna donde pastean camélidos. El patrón de asentamiento de la zona de valle se caracteriza por ser nucleado, mientras que el de la puna se caracteriza por un hábitat disperso con pequeños caseríos de 2 o 3 casas intercomunicadas y rodeadas de tierras de pastoreo. Los niños que viven en estos caseríos en mayoría no poseen libros y si los tienen son muy pocos, tampoco tienen acceso a películas, ni a la televisión ni a las computadoras. El nivel de instrucción de los padres es inferior al de las muestras de los estudios realizados en los EE.UU., cuyos padres tienen por lo menos garantizada la formación escolar hasta el quinto grado de primaria.

En este estudio podremos examinar si el uso de las proposiciones genéricas se extiende más allá de los contextos didácticos de la clase media o si son también elaboradas y comprendidas en comunidades en las que los padres tienen poca educación formal y poco contacto con libros. El uso de enunciados genéricos está muy generalizado en las conversaciones cotidianas entre padres e hijos tanto en los EE.UU. como en China, lo que hace suponer que la expresión de los genéricos es un fenómeno transcultural. Por otra parte, las poblaciones donde se han llevado a cabo los estudios previos sobre genéricos se caracterizan por ser relativamente instruidas, en zonas urbanas o periféricas y en contacto directo con los libros, la televisión y diarios. Este estudio proporciona información sobre contextos culturales y económicos muy diferentes de los ya estudiados.

Para analizar la función de las oraciones genéricas en el desarrollo conceptual temprano, es necesario establecer las representaciones que se hacen los quechuahablantes de las proposiciones genéricas, tanto las de los niños como las de los adultos. Esto debe incluir 1) la relación entre las oraciones genéricas y las que no lo son (es decir, las oraciones específicas). Para esto, llevamos a cabo dos estudios experimentales (empíricos, expuestos en las secciones siguientes de este artículo). El primer experimento consistió en una comparación entre las interpretaciones de oraciones genéricas (ej., Llamaq millman yuraqchu?; «Las Ilamas itienen lana blanca?») y los cuantificadores «todos» y «algunos» (ej. Llapa Ilamaqchu millman yuraq?; «iTodas las llamas tienen lana blanca?», Wakin Ilamaqchu millman yuraq?; «¿Algunas llamas tienen lana blanca»?). El segundo experimento consistió en comparar oraciones genéricas (ej. «Urrrr» nispa nin khuchiqa; Los cerdos dicen «urrr») con oraciones específicas (K'ayra manaña tusunchu; «La rana ya no está bailando»). Estos experimentos han sido expuestos en detalle en una publicación previa (Mannheim et al., 2011). Reenviamos al lector a esta publicación para detalles adicionales sobre las fuentes, los procedimientos y el análisis. 


\section{1. Experimento $n .^{\circ} 1$ : respuestas afirmativas/negativas respecto a oraciones genéricas y con cuantificadores}

En este experimento el grupo de participantes estaba constituido por 118 quechuahablantes de 3 a 90 años de edad, habitantes de una comunidad rural de la provincia de Calca (Cusco) y divididos en 5 grupos: 3-6 años; 7-9 años; 10-12 años; 14-35 años y 36-90 años. Los participantes más jóvenes no estaban aún escolarizados y muchos de los otros estaban en contacto con la lengua española en mayor o menor grado; sin embargo toda la investigación se llevó a cabo completamente en quechua, lengua exclusiva de comunicación en la comunidad. Cada participante fue entrevistado individualmente con una serie estándar de preguntas $\mathrm{SI} / \mathrm{NO}$, diseñadas con el fin de evaluar la comprensión que los participantes tenían de los enunciados genéricos y, con fines de comparación, la de los cuantificadores «todos» (Iliw o llapa) y «algunos» (wakin). Nuestro principal objetivo era comparar el espectro de los enunciados genéricos con el de los cuantificadores. Partimos del supuesto que el cuantificador «todos» abarcaría un espectro más amplio (refiriéndose a todos los elementos de una clase), que las proposiciones genéricas abarcarían de alguna manera un espectro más restringido (haciendo referencia a varios o muchos elementos de la clase, pero no necesariamente a todos) y que «algunos» abarcaría un espectro más restringido aún (refiriéndose incluso solo a pequeños subgrupos de la clase). En efecto, estudios previos con hablantes de lengua inglesa mostraron que hacia los 4 años el enunciado «algunas flores son amarillas» era aceptado con más frecuencia que el enunciado «las flores son amarillas», y este mucho más que el de «todas las flores son amarillas» (Hollander et al., 2002). Si bien es cierto que las diferencias entre los enunciados genéricos y los cuantificadores no solo se realizan en el ámbito del predicado (véase Cimpian et al., 2010) este constituye un elemento importante de diferenciación entre ellos.

En este experimento hemos dado a los participantes los mismos cuestionarios utilizados previamente con hablantes de lengua inglesa, con los enunciados adaptados al contexto sociocultural de los Andes. Por ejemplo, la pregunta realizada en contexto de habla inglesa «之ेlos osos tienen piel blanca?», fue reemplazada en el contexto andino por «ìlas llamas tienen piel blanca?». Los participantes escuchaban tres variantes de la frase nominal sujeto: genérico (ej. Llamaq millman yuraqchu?; «ilas llamas tienen piel blanca?»), «todo» (Llapa Ilamaqchu millman yuraq?; itodas las llamas tienen piel blanca?), y «alguno» (Wakin Ilamaqchu millmallan yuraq?; «ialgunas Ilamas tienen piel blanca?»). Hubo también tres tipos de propiedad: una de espectro amplio (afirmativo para todos o casi todos los elementos de la categoría; por ejemplo Wawa sinqayuqchu?; «ìlos bebés tienen nariz?»), otra de espectro reducido (cierto solo para un subgrupo de la categoría; por ejemplo, Llamaq millman yuraqchu?; «之ेlas Ilamas tienen piel blanca?» o Sumbreru watanayuqchu?; «之̇los sombreros tienen atados?») y propiedades generales anómalas o sin sentido (que no se aplican a ningún miembro de la categoría; por ejemplo, Atuq riluqniyuqchu?; «żlos zorros tienen 
reloj?»). Nuestro interés principal estaba centrado en las propiedades generales de los ítems con espectro reducido porque era entre los ítems con espectro reducido que hemos hipotesiado el mayor grado de diferenciación en expresión. Los de espectro más amplio son también interesantes porque deberían mostrar la distinción entre «todos» y genérico por un lado y «alguno» por otro lado ya que en quechua «alguno» (wakin) es contrastivo e implica «no todos». Por último, algunas expresiones anómalas fueron introducidas para asegurarnos que algunas preguntas tendrían una respuesta negativa univoca (con el fin de evitar que hubiese una inclinación a contestar todos los ítems con la misma respuesta). Ambos factores (propiedades y expresiones) se cruzaron produciendo nueve clases de ítems. En total los participantes escucharon 4 ítems de cada tipo, con un total de 36 ítems por participante.

Estos resultados mostraron que se presentan efectos importantes entre la expresión (genérico, todo, alguno), las propiedades (espectro amplio, reducido, anómalo) y los grupos de edad (3-6, 7-9, 10-12, 14-35 y 36-90). Empecemos con el patrón obtenido sin distinguir entre los grupos de edad. En quechua de manera general, la interpretación de las expresiones genéricas es similar a la de los hablantes de otras lenguas (ingles y mandarín), en el sentido en que los genéricos se distinguen de los cuantificadores «todo» $y$ «alguno». Los participantes distinguen entre las expresiones genéricas y las que con el cuantificador «alguno» cuando toman en cuenta las propiedades de espectro amplio (todos están de acuerdo en que los bebes tienen nariz y que todos tienen nariz, pero niegan que «algunos bebes tengan nariz»), distinguen también los genéricos del cuantificador «todo» cuando consideran las propiedades de espectro estrecho (con frecuencia están de acuerdo en que las llamas sean blancas y que «algunas» Ilamas lo sean también, pero niegan que todas las llamas sean blancas). En otras palabras, los genéricos funcionen como «todo» cuando se trata de propiedades de espectro amplio y la función como «alguno» cuando se trata de propiedades de espectro estrecho.

Estos patrones sobre las propiedades de espectro amplio se encontraron en todos los grupos de edad incluyendo a los niños más pequeños. Es así que por ejemplo los niños quechuahablantes entre tres y seis años tienden a responder con más frecuencia de manera afirmativa a las preguntas «ilos bebes tienen nariz?»o «itodos los bebes tienen nariz?» que a la pregunta «żalgunos bebes tienen nariz?». Por lo tanto podemos decir que uno de los aprendizajes precoces en la comprensión de los enunciados genéricos supone que las propiedades se encuentren ampliamente distribuidas al interior de la categoría y no se limiten solo a un pequeño subgrupo. Respecto a las propiedades específicas (ej. ¿Las llamas son blancas?), encontramos diferencias deslumbrantes en las respuestas de acuerdo a la edad. Mientras que los patrones de aprobación fueron relativamente estables para los genéricos y para el cuantificador «todo», se registró un incremento significativo en el grupo de nueve a quatorce años para las preguntas con el cuantificador «alguno». Mientras que los niños más pequeños (de tres a nueve años) respondieron a los ítems de propiedades estrechas de la misma manera independientemente de las expresiones, sin diferenciar los genéricos de los cuantificadores «todos» y 
«algunos», todos los más grandes se lo diferenciaban de algún grado. Para el grupo de diez-doce años, los genéricos presentaban un mayor grado de aprobación que el cuantificador «todo»; para el grupo de 14-35 años, las tres expresiones mostraban diferencias significativas: «alguno» tenía el mayor índice de aprobación, seguido por los genéricos, con «todo» el más bajo; y para los participantes mayores (entre 36-90 años), «alguno» tenía un mayor índice que «todo», con los genéricos entre los dos (pero sin distinguirse de ambos de manera significativa). Por lo tanto, podemos observar que cuando se toma en consideración las propiedades de espectro estrecho la distinción entre los genéricos y los cuantificadores «todos»y «algunos» aparece tardíamente. Por último, como era de esperarse, las condiciones de expresión no ejercieron ningún efecto en los ítems anómalos, que fueron rechazados en todos los casos; es así que nos concentramos exclusivamente en los ítems de espectro amplio y reducido.

De manera general, de los resultados del experimento uno se desprenden tres conclusiones principales:

1) los quechua hablantes distinguen los conceptos genéricos de los cuantificadores «todo» y «alguno»;

2) al principio las construcciones genéricas son concebidas como abarcando toda la clase (como el cuantificador «todo»); sin embargo los niños aceptan que puedan haber excepciones (como el cuantificador «alguno»);

3) el desarrollo cognitivo involucrado en esta tarea incluye cambios en la comprensión de los cuantificadores; en cambio la comprensión de los enunciados genéricos permanece sorprendentemente estable e inalterable a lo largo del desarrollo cognitivo.

\section{2. Experimento $n .^{\circ} 2$ : interpretación semántica de las construcciones genéricas y no genéricas}

En este experimento se analizaron dos factores que consideramos podrían ser utilizados por los hablantes para determinar si un enunciado es genérico. El primero es la ausencia de marcadores lingüísticos de especificidad. Partimos de la hipótesis que en lengua quechua, como en otros idiomas, los genéricos constituyen una condición base (default) caracterizada por la ausencia de información que indica la especifidad (Gelman \& Brandone, 2010). En otros términos, partimos de la hipótesis que las construcciones genéricas son no marcadas en términos lingüísticos. De ser cierto, esto sería importante por dos razones: primero, resolvería el enigma de cómo los niños adquieren los genéricos, tomando en cuenta que estos se expresan de manera variable y carecen de un marcador único; segundo, apoyaría la afirmación que los genéricos son también el modo de generalización de base en términos conceptuales (Leslie, 2008).

El segundo factor que hemos examinado ha sido la animacia. Los estudios hechos hasta ahora hacen suponer que el uso de las expresiones genéricas se privilegia dentro del dominio cognitivo «animal». Es así que al momento de 
tomar en cuenta objetos tantos conocidos como desconocidos, los hablantes de lengua inglesa tienen una mayor tendencia a producir construcciones genéricas refiriéndose a los animales que a los artefactos. La razón exacta de esto no queda claro, pero muy probablemente tiene que ver con nuestras teorías tácitas (naive theories) sobre los animales (a quienes le atribuimos una capacidad de acción, con complejas propiedades inherentes compartidas por todos los miembros de la categoría) versus las teorías sobre los artefactos (los cuales no comparten el mismo estructura de clase natural (natural kind). En el segundo experimento quisimos ver si este fenómeno se repetía en un contexto cultural distinto (una comunidad rural indígena con muy pocos artefactos), y si se llevaba a cabo en la realización de una tarea distinta (la interpretación de una oración, en vez de la elaboración de la misma).

El grupo de participantes de este experimento estaba constituido por 80 quechuahablantes, entre nueve y 90 años de edad, divididos en tres grupos (nuevedoce, 14-35, y 36-90). Todos eran de la misma comunidad que los del primer experimento y muchos de ellos habían participado también en un estudio previo. Tomando en cuenta que la tarea era más exigente que la del primer experimento, nos concentramos exclusivamente en los tres grupos mayores de edad.

Un estudio previo sobre los locutores de mandarín sirvió de modelo para este experimento (Gelman \& Tardif, 1998: estudio 3). Los participantes tenían que escuchar una serie de dieciseis oraciones con una base nominal que variaba respecto a su animicia (ej.: «los toros» eran animados; «un tractor» inanimado) y en cuanto a su carácter genérico. Las oraciones con un exponente gramatical de especificidad (p.e. un tiempo verbal pasado, un imperativo, un durativo) fueron consideradas no genéricas; el resto, que no presentaban marcado la especificidad, fueron consideradas como genéricas.

La tarea consistía en decidir para cada oración si la frase nominal hacía referencia a una instancia (huq), a casi todas (yaqa llapa), o solamente a algunas (wakillanchu). Muchos participantes dieron respuestas que no correspondían a ninguna de las tres alternativas propuestas (ej. «todos», «muchos», «algunos», «pocos»). Las respuestas «casi todos», «muchos» y «todos» fueron consideradas como genéricas; todas las demás no lo fueron.

A través de todo el corpus, los resultados fueron evidentes y precisos. Los quechuahablantes interpretan de manera inmediata como genéricas las frases que carecen de especificidad, y además tienen mayor tendencia a interpretar las frases nominales animadas como genéricas. Estos resultados muestran que las interpretaciones genéricas son para los quechuahablantes la interpretación base (default interpretation) y que la tendencia de asociar lo genérico a lo animado depende del contexto social y lingüístico. 


\section{CONCLUSIONES}

Ambos experimentos confirman que durante la infancia los niños quechuahablantes diferencian los enunciados genéricos de los específicos (experimento $n .^{\circ} 2$ ) así como los cuantificadores (experimento n. ${ }^{\circ} 1$ ). Es así que las construcciones genéricas, tanto para los quechuahablantes como para los de lengua inglesa y mandarín, representan un mayor espectro referencial que los enunciados específicos; no obstante estos difieren del significado lógico y preciso de «todo» y «alguno». Estos ocupan el mismo espacio semántico, lo cual permite a los locutores expresar las generalizaciones con relevancia a los tipos semánticos a la vez que permiten las excepciones. Además, los quechuahablantes atribuyen un valor genérico de manera más inmediata a las frases nominales con agentes animados que a las frases con agentes inanimados. Lo que sorprende de estos resultados es la manera en la que estos coinciden con los resultados previos obtenidos con los hablantes de inglés y de mandarín. Podemos observar notables y fuertes coincidencias en la interpretación de los genéricos por parte de los hablantes de distintas lenguas y complejos culturales a través del mundo.

Otro resultado sobresaliente es el hecho de que los hablantes de quechua atribuyen un valor genérico a los enunciados que lingüísticamente están no marcados (unmarked). Los enunciados que carecen de marcas lingüísticas de especificidad (incluyendo morfemas gramaticales así como marcas lexicales independientes) tuvieron mayor tendencia a ser interpretados como genéricos que aquellos que poseían hasta un solo morfema gramatical. Este resultado es notable porque confirma la hipótesis según la cual las construcciones genéricas constituyen la interpretación lingüística no marcada.

Por último, constatamos que incluso los locutores de quechua más jóvenes hacen la diferencia entre los genéricos y el cuantificador «algunos» ya que «algunos\} (wakin) en quechua tiene un significado exclusivo («no todos»). Este resultado es de gran interés sobre todo porque los hablantes más jóvenes de inglés y de mandarín no distinguen los genéricos de «algunos».

De una forma más general, consideramos que estos resultados muestran la clásica relación entre las capacidades cognitivas universales y las particularidades del input de una lengua específica. Confirmamos que la comprensión de los genéricos sería imposible sin una distinción conceptual previa entre el individual y el tipo. Tal distinción conceptual es necesaria para atribuir el estatus «no marcado» - $\mathrm{O}$ de base- a los conceptos genéricos, cuando los aprendices no cuentan ni con la evidencia de marcadores lingüísticos o instancias de la vida real (después de todo, no se puede enumerar o señalar a las llamas en general — solo a llamas particulares-). De igual manera, la variabilidad translingüística de las formas en que las construcciones genéricas son expresadas muestra que los aprendices deben asimilar tipos y patrones de indicadores distintos para discernir la manera en la que las distinciones conceptuales se expresan en la lengua que están aprendiendo. Más aún, los matices de la lengua que están aprendiendo añaden diferencias sutiles en los valores semánticos adquiridos. 
Los conceptos aprendidos de manera espontánea, aprendidos pero no enseñados, recurren a principios ontológicos básicos que, a su vez, estructuran las teorías tácitas al interior de las cuales el desarrollo de los conceptos se lleva a cabo. De la misma forma, el desarrollo cognitivo se nutre de los recursos morfosintácticos de una lengua específica, que vehiculan conceptos relevantes y que los asignan a un domino cognitivo específico. Las construcciones genéricas, aunque no tengan marcas morfológicas, constituyen un elemento esencial de articulación entre los procesos cognitivos universales y las especificidades de las lenguas y las culturas, la matriz del armazón y ontología específicamente quechuas.

\section{Referencias citadas}

CAREY, S., 1985 - Conceptual change in childhood, xiv + 226 pp.; Cambridge, MA: MIT Press.

CARLSON, G. N. \& PELLETIER, F. J. (eds.), 1995 - The generic book, x + 463 pp.; Chicago: University of Chicago Press.

CHIERCHIA, G., 1998 - Reference to kinds across languages. Natural Language Semantics, 6: 339-405.

CIMPIAN, A. \& MARKMAN, E. M., 2008 - Preschool children's use of cues to generic meaning. Cognition, 107: 19-53.

CIMPIAN, A. \& MARKMAN, E. M., 2009 - Information learned from generic language becomes central to children's biological concepts: Evidence from their open-ended explanations. Cognition, 113: 14-25.

CIMPIAN, A., GELMAN, S. A. \& BRANDONE, A. C., 2010 - Theory-based considerations influence the interpretation of generic sentences. Language and Cognitive Processes, 25 (2): 261-276.

CSIBRA, G. \& GERGELY, G., 2009 - Natural pedagogy. Trends in Cognitive Sciences, 13: 148-153.

CUSIHUAMÁN GUTIÉRREZ, A., 1976 - Gramática quechua, CuzCo-Collao, 299 pp.; Lima: Instituto de Estudios Peruanos.

FALLER, M., 2002 - The semantics and pragmatics of evidentials in Cuzco Quechua; Stanford: Stanford University. Tesis doctoral en lingüística.

GELMAN, S. A., 2003 - The essential child: Origins of essentialism in everyday thought, $\mathrm{x}$ + 382 pp.; New York: Oxford University Press.

GELMAN, S. A., 2004 - Learning words for kinds: Generic noun phrases in acquisition. In: Weaving a lexicon (D. G. Hall \& S. R. Waxman, eds.): 445-484; Cambridge, MA: MIT Press.

GELMAN, S. A. \& BRANDONE, A., 2010 - Fast-mapping placeholders: Using words to talk about kinds. Language Learning and Development, 6: 223-240.

GELMAN, S. A. \& MARKMAN, E. M., 1986 - Categories and induction in young children. Cognition, 23: 183-209.

GELMAN, S. A. \& TARDIF, T. Z., 1998 - Generic noun phrases in English and Mandarin: An examination of child-directed speech. Cognition, 66: 215-248.

GELMAN, S. A. \& WELLMAN, H. M., 1991 - Insides and essences: Early understandings of the nonobvious. Cognition, 38: 213-244. 
GELMAN, S. A. \& WILLIAMS, E., 1998 - Enabling constraints for cognitive development and learning: Domain specificity and epigenesis. In: Cognition, perception and language. Vol. 2. Handbook of Child Psychology (Fifth Ed.) (D. Kuhn \& R. S. Siegler, eds.): 575-630; Nueva York: Wiley: W. Damon, Editor-in-Chief.

GELMAN, S. A., WARE, E. A., MANCZAK, E. M. \& GRAHAM, S. A., 2013 - Children's sensitivity to the knowledge expressed in pedagogical and nonpedagogical contexts. Developmental Psychology, 49 (3): 491-504.

GREENBERG, J. H., 1963 - Some universals of grammar with particular reference to the order of meaningful elements. In: Universals of language (J. Greenberg ed.): 73-113; Cambridge, MA: MIT Press.

HAUF, P., PAULUS, M. \& BAILLARGEON, R., 2012 - Infants use compression information to infer objects' weights: Examining cognition, exploration, and prospective action in a preferential-reaching task. Child Development, 83: 1978-1995.

HOLLANDER, M. A., GELMAN, S. A. \& STAR, J., 2002 - Children's interpretation of generic noun phrases. Developmental Psychology, 38: 883-894.

KEIL, F., SMITH, W., SIMONS, D. \& LEVIN, D., 1998 - Two dogmas of conceptual empiricism: Implications for hybrid models of the structure of knowledge. Cognition, 65: 103-135.

LESLIE, S. J., 2008 - Generics: Cognition and acquisition. The Philosophical Review, 117: 1-49.

MANNHEIM, B., GELMAN, S. A., ESCALANTE, C., HUAYHUA, M. \& PUMA, R., 2011 - A developmental analysis of generic nouns in Southern Peruvian Quechua. Language Learning and Development, 7: 1-23.

MASSEY, C. \& GELMAN, R., 1988 - Preschoolers' ability to decide whether a photographed unfamiliar object can move itself. Developmental Psychology, 24 (3): 307-317.

OPFER, J. E. \& GELMAN, S. A., 2010 - Development of the animate-inanimate distinction. In: Wiley-Blackwell handbook of childhood cognitive development (U. Goswami, ed.): 213-238; Malden, MA: Wiley-Blackwell.

SIMONS, D. \& KEIL, F. C., 1995 - An abstract to concrete shift in the development of biological thought: the insides story. Cognition, 56: 129-163.

SLAUGHTER, V. \& GOPNIK, A., 1996 - Conceptual coherence in the child's theory of mind: Training children to understand belief. Child Development, 67: 2967-2988.

TARDIF, T., GELMAN, S. A., FU, X. \& ZHU, L., 2012 - Acquisition of generic noun phrases in Chinese: Learning about lions without an '-s'. Journal of Child Language, 30: 1-32.

WAXMAN, S. R. \& GELMAN, S. A., 2010 - Different kinds of concepts and different kinds of words: What do words do for cognition? In: The making of human concepts (D. Mareschal, P. Quinn \& S. Lea, eds.): 99-129; Oxford: Oxford University Press.

WELLMAN, H. M. \& GELMAN, S. A., 1998 - Knowledge acquisition in foundational domains. In: Cognition, perception and language. Vol. 2. Handbook of Child Psychology (Fifth Ed.) (D. Kuhn \& R. S. Siegler, eds.): 523-573; Nueva York: Wiley W. Damon, Editor-in-Chief.

XU, F. \& KUSHNIR, T., 2013 - Infants are rational constructivist learners. Current Directions in Psychological Science, 22 (1): 28-32. 